\title{
THE CONVERGENCE OF ART AND SCIENCES IN DIGITAL AGE: SOME PERSPECTIVES FROM NYAWA EXHIBITION IN MALAYSIA.
}

\author{
Mohd Nasir Baharuddin ${ }^{1 *}$, Adam Mohd Salehuddin ${ }^{1}$, Faridah Qamaruz Zaman ${ }^{2}$ and \\ Ahmad Shukri Muhammad Noor ${ }^{3}$ \\ ${ }^{I}$ Faculty of Design and Architecture, University Putra Malaysia \\ ${ }^{2}$ Faculty of Science, University Putra Malaysia \\ ${ }^{3}$ Faculty of Engineering, University Putra Malaysia
}

\begin{abstract}
This article tries to introduce the idea of art and science into new contemporary aesthetics in the contexts of digital age in Malaysian art. This aesthetic field of investigation has led to a reassessment of representation based on Nature's Yield and Wonders of Art (NYAWA) exhibition. The exhibition process will be structured by using 'visual and discourse analysis' methodology where the process of information and visual data will be shaped and investigates pertaining to the integration of art and science and how the exhibition materials and the language can be intersect. As a result, it tries to open a new gap of various disciplines in producing new ideas which enhance more dialogs and avenues between art and science as a creative convergence. Hopefully, this paper will try to operate an innovative and engaging manner, in order to support the development of new interpretive methodologies among scientists and artists to gives new ideas for future artistic creation. As a result, it can intrigue new directions for the visual arts industries. Indeed, search for balance to the convergence of science and art. Furthermore, this convergence can foster the interdisciplinary and collaborative work that is essential in building New Malaysian Contemporary Art.
\end{abstract}

Keywords: Creative Convergence, Interaction, Across Disciplinary

\section{Introduction}

The aim of this article is to redefine art in creative ways at the interdisciplinary visibility where scientific inquiry and new artistic tecnologies shapes the aesthetic values and communication culture, either local or global. Perhaps these intergrations needed a new way of directions for future artistic creation in a rapidly changing world where the boundaries between art and science are becoming transparent and merged. The changes of technology have a significant impact on the culture of human society. The way digital media is always compressed in our minds to stand up and face new cultures to improve our social lives. The competition between science, technology and art stands as new-environment that allows us to see a variety formation of the highest intellectual accomplishments. Many new technologies in the $21^{\text {st }}$ century are centred on self-extension, driven by high-speed internet, the proliferation of mobile devices and computer gaming. This also embraces a significant role for the intersection between the arts, technology and science. From an album of self to an instagram selfie, from a locational geo-tagging device to a new world of medicines, and our lives extend both outwards into space through many different forms and also extend into time by being able to live so much longer than humans have just a century ago. Naturally, ideas and concepts of the self are being revised in the $21^{\text {st }}$ century.

\footnotetext{
"In the $21^{\text {st }}$ century, some of the most dynamic works of art are being produced not in the studio but in the laboratory, where artists probe cultural, philosophical and social questions connected with cutting-edge scientific and technological research. Their works ranges across disciplines microbiology, the physical sciences, information technologies, human biology and living systems, kinetics and robotics - taking in everything from eugenics and climate change to virtual reality and artificial intelligence" (Stephen Wilson, 2010).
} 
However, the creation of artistic form through this integration has led to alternative media conflicts in various disciplines and perspectives that deal with new study and critical visual theories. This growing and global movement has taken on an increasingly collaborative socio cultural and scientist activist as well as a visual artist, to address the world's problems and will require creative and inspiring collaborations between people, places and creatures. So, in order to engage and cooperating an exciting activity in creating a different set of approach, future artistic creation will have to change the visibility and methodological approach.

The combination of art and science urgency gives a new way to see the world. Derived from artistic experiment and lab culture, it is able to create aesthetic, emotional, environmental, educational and social values. Reality towards culture in the context of art seems to change and build new existence and more nuanced understanding to educate people about the role of art itself. The presence of theoretical in science adapted by these artists was opened a new boundary and create an alternative space to be referred as contemporary art in Malaysia. The existence of new syllabuses and programme in media applications will have a more open and global impact in assessing works of art in today's digital era.

Anyway, digital age is not only about creating technologies with new gadgets and media.

"They are radically transforming basic philosophical ideas about the nature of physical world, time and space, the nature of life and intelligence, and the limits in our abilities to transform the world and humanity. The technological is intertwined with attitude, value, culture and socio political economic environments". (Stephen Wilson, 2008).

It reflects and compressed in our minds to stand up and face new dimension of thinking and existence to improve our social lives and make things beautiful. As mentioned by Einstein, a Physicist: "The most beautiful thing we can experience is the mysterious. It is the source of all true art \& science". Also by James Mae.C, an astronaut: "Science provides an understanding of a universal experience and art provides a universal understanding of a personal experience", so....neither science nor the arts cannot be complete without combining their separate strength. This also be mentioned by biologist, E. O. Wilson, describes intuitively: "Science needs the intuition and metaphorical power of the arts and the arts need the fresh blood of science". (Einstein, A., Mae. C. J., and Wilson. E, O., 2018)

\begin{abstract}
"Since science and art come from what are thought to be opposite ends of the spectrum of human understanding, there seems to be an added energy released when they embrace. Whatever the reason, it is tempting to conclude that contemporary science and art have found gaps in each other that require filling”. (Michal Giboda,. 2005)
\end{abstract}

So, in order to fill the gap, every disciplines of knowledge have to come forward and develop new energy of environment for the artistic productions to grow. Although, this topic is not something new in this era because in the world right now, all things had already been invented, but someone can make a shift by looking at other disciplines to create something new. At this time, all creative creations will have to change into new system of methodologies which can develops further exploration that can enhance the scope of knowledge with difference perspective and communication.

\title{
Nature's Yield and Wonders of Art (NYAWA) exhibition
}

It is an annual exhibition that was start up in 2012. The aim is basically to represent and enhance all research's discipline into the idea of representational. This activity will try to connect all sorts of research discovery and discipline in creating new dimension space reality. To exposed and inculcate the laboratorial process to visual communication and intersection. This exhibition was derived from various disciplines of studies, where it came from faculty of design and architecture, language, biotechnology, engineering, marine, agriculture, veterinary, education and medical. 
Presenting NYAWA into an exhibition is the most challenging work, especially how to transmit the subject of the study into a visually setting. It needs a lot of interaction process among participants and how to merge other disciplines into a certain level of agreement. In fact, curating NYAWA brings an attitude that acknowledges a dialogical interaction within the mysterious and factual discovery about natural and artistic creation. It stimulates the reality of an object as element of information and the spirit of natural pattern and its behaviour.

\section{Exhibition Objective}

Major purpose in creating this exhibition is to merge and integrate artists and scientist to create an independent zone, based from research - where all participants will integrating all communication commentary with the search for the new knowledge and the elaboration of new technical possibilities. In addition it gives four major significances:

- Amplifying thought: a concept development - Acknowledge the process of thinking and ideas.

- Sharing consciousness: collaborative processes - the spirit of working together and building a knowledge solution.

- - Seeding structure: self-organizing systems - to plant the system of values between self and others.

- Making metaphors: knowledge navigation - to build new understanding from knowledge process that produce meaning and the benefit of the research.

In this case, the productions of the exhibition function as a question, process of knowledge, documentation, and to build communication and interaction among the participants and public. It is an element of one field to explore and share with difference knowledge in creating new experience. Even it can be a map of a potential approach in an alternate field and perspective, by describing the process and research activities. In addition, the production can function as a database of ideas that enable differing people to access. Bringing an approach to particular question that science will not allow.

In order to intersect the dialogue and explore these two disciplines, the players have to intergrate the language of a visual expression and the language of empirical data into a visual sign of codes - it needs to transmit information based on the culture as 'technoculture'.

"A culture as informed or defined by its technological activity, especially a culture characterized by a high level of technological development; (also) the practices, attitudes, etc., characteristic of those proficient in the use of information technology". (Oxford living Dictionaries).

It tries to bring scientists and artists to become innovators in the development of emerging technologies. By emerging the research discipline, one's can become the core of new cultural trends and industries, in order to demonstrate and adept with the current pace of change.

\section{Methodology}

The strategies of the programme will be structured by using 'visual and discourse' methodology where the process of information and visual data will be shaped and investigates through social production of meaning. It goes by textual and the roots of the subject pertaining to the integration of art and science. The method will focus on how the interdisciplinary production of knowledge be conducted...and how the exhibition materials and the visual language can be intersect and apply. 
Discourse is concerned with a critical analysis of the use of language and the reproduction of belief systems, in discourse - defined by a group of ideas or patterned way of thinking which can both be identified in textual and verbal communications and located in wider social structures.

"By adding a linguistic approach to an understanding of the relationship between language and ideology, exploring the way in which theories of reality and relations of power are encoded in such aspects as the syntax, style and rhetorical devices used in texts”. (Deborah Lupton. 1992)

Therefore in order to prepare for the exhibition, here are some work steps that will help to conduct a systematic discourse analysis methodology.

\section{Establish the context and issue}

Firstly by looking at the big picture based on text and talk. Find out whether the sources are responses to any contexts. Set an open discussion, by slowing down the ego and emotion, slowing down the intellect, and create as a receiver and reflective mode among participants. Figure out the issue and the subject of research topic in order to start a communication process.

\section{Explore the production process}

Try to figure out the background of the research study. Tackle the research subject, medium and the genre what they are working with. Frame the meaning of the actual text and any additional features of the medium that might contribute to or shape meaning of the production. What kind of genre one's source belongs to, that will later help to get its message and production across?

\section{Prepare and coding the material for analysis}

Looking for text - statement, content and image, the visual materials such as photo, specimen. Object - as material form which derived from the empirical data. Coding the data, such as paragraphs, sentences, or individual words, pictures, symbols, links, or articles. Coding the categories. Based on the kind of question you are asking, and your knowledge of the subject matter, you will already have a few key themes in mind that you expect to find,

Write down topics that you think might be related to these key themes. These factors are the basis for starting categories.

\section{Examine the structure of the text}

How to deal with one discourse? Does it first make a counter-factual case, and make the main argument? Identify how the argument is structured: does the text go through several issues one by one? Try to be selective. How to visualise the text/subject into a language of visual and product of presentation.

\section{$\underline{\text { 5. Collect and examine discursive statements }}$}

Once you have a good idea regarding of the text, you can zoom in on the individual statements, or discourse fragments. A good way to do this is to collect all statements with a specific code, and to examine what they have to say on the respective discourse strand. This collection of statements will allow you to map out what "truths" the text establishes on each major topic. 


\section{Identify key concept and cultural references}

Established what the context of source material is in relation to cultural contexts. Now think about how the context informs the argument. Does your material contain references to other sources, or imply knowledge of another subject matter? What meaning does the text attribute to such other sources? Exploring these questions will help you figure out what function intertextuality serves in light of the overall argument until it achieves certain agreement.

\section{Identify visual linguistic and representational mechanisms}

Identify how the various statements function at the level of visual language and presentation.

- Visual features: what the subjects and objects of the visual.

- Visual formation: technical and skill, object and space.

- Sign and symbol: marks, an image, text as clue as sign and metaphor

- Material and message: the presentation/production, for example an installation,

- Image projection such as video and graphic text.

\section{Interpret the data and start building a statement and presentation.}

What does it all mean? How to build the interpretation and the content of knowledge? How to position on the topic you examined and the situation. How do their arguments draw from and commonly accepted in the place that this argument was made? Until it can achieves certain agreement and benefit from the discourse? It is time to get your results across to your target audience. What is interesting about the production and the visual presentation? How the production reflects to the audience? The impact of the production and interaction? And how the work can build a communication systems and processes.

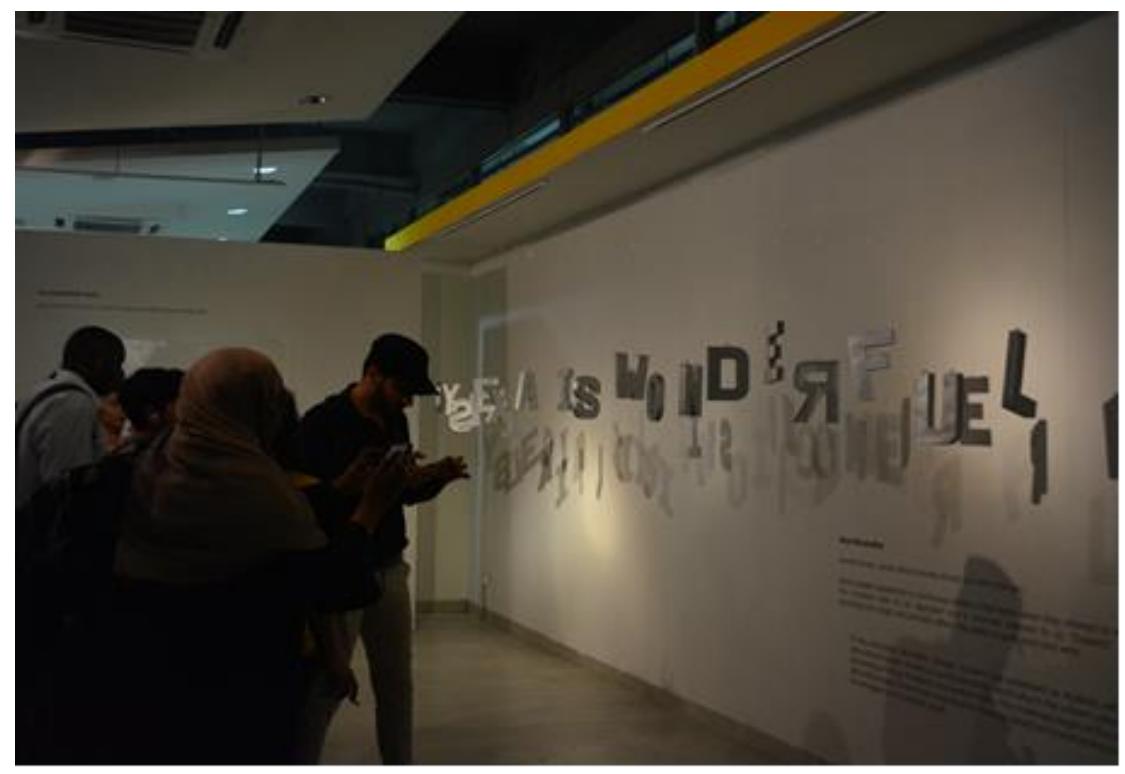

Figure 1: Word Illumination,

Samsilah Roslan, Zeinab Ghiami, Noorlila Ahmad and Jinimy Dangko (2016).

In Figure 1, an installation work that consist a word 'dyslexia is wonderful', hanging and rotating with shadow casting. The installation creates a 'paradox' of perception in reading the words. Some people experience a 
continuous battle in their brain when they attempt to read. This condition akin to an alphabetical war is normally referred as "Dyslexia". It is neurological in origin and primarily affects the ability to spell, read and write. Literally, it means difficulty with words. Dyslexia sufferers also known as dyslexics are highly gifted and talented individuals. They learn like other students but with more focus on letter and word recognition. Dyslexia is usually hereditary; and it affects the sufferer's phonological processing of strings of objects and symbols. The challenge begins at the process of decoding single words to form sounds and meanings (read) and transforming sounds into an arrangement of symbols (spell). Nevertheless, the sufferer does not have a problem with the comprehension of meaning.

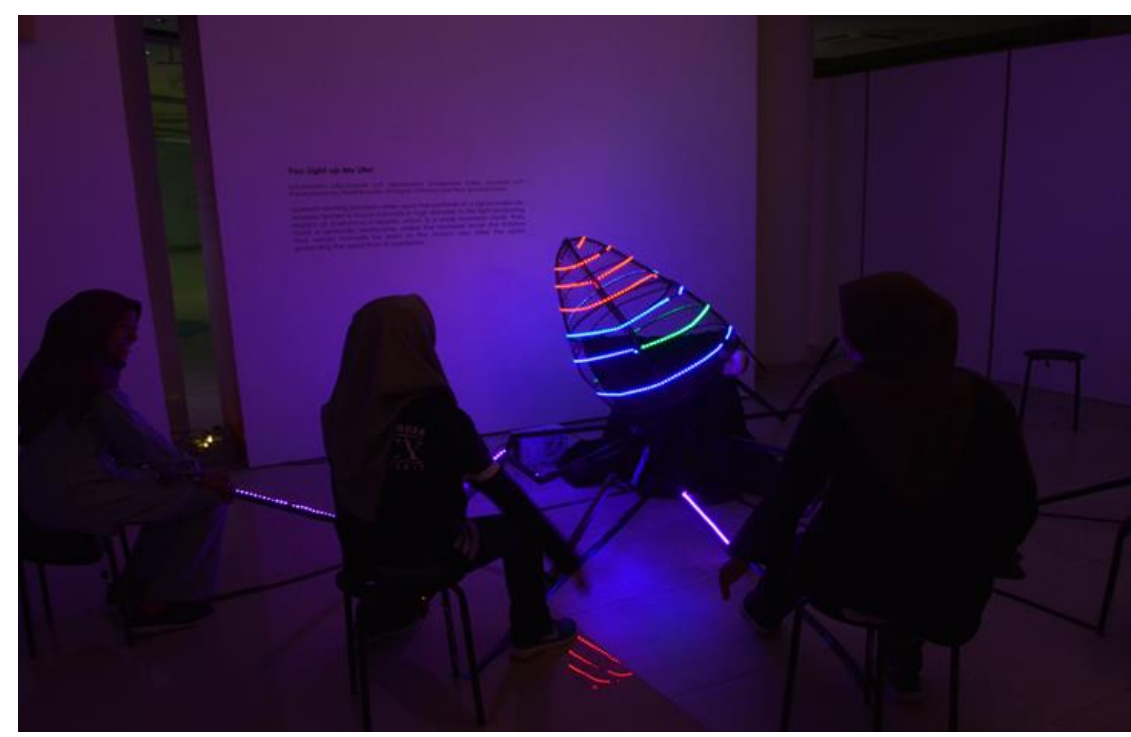

Figure 2, You light up my life!

Suruthimitra Okpoluaefe A/P Okpoluaefe Godspower Udezi, Kavesha A/P Parameswaran, Hanif Baharin, Siti Sarah Othman and Wan Zuhainis Saad (2017)

As seen in Figure 2, it is an interactive installation artwork that used LED and censor. The main issue of this creation is about misconstrued that communication can only be done by macro-organisms including the common man. This is the central dogma that is circulated universally. Organisms like Alivibrio fischeri, or what was once known as vibrio fischeri has challenged this theory. Microorganisms are able to communicate through a mechanism called quorum sensing. Quorum sensing is a system of stimuli and response correlated to population density. Quorum sensing allows bacteria to restrict the expression of specific genes to the high cell densities at which the resulting phenotypes will be most beneficial. They coordinate gene expression according to the density of their local population. This is exactly how Alivibrio fischeri showcases its light producing abilities.

The regulation of the gene expression of Alivibrio Fischeri changes in response to cell density. Quorum sensing bacteria produce and release Autoinducers. Autoinducers modify gene expression after a sudden change in cell population density. Quoram sensing bacteria relies upon the synthesis of a signal molecule. Alivibrio fischeri is found naturally in high densities in the light producing organs of Euprymna scolopes, which is a small Hawaiian squid. They have a symbolic relationship where the microbes erase the shadow that would normally be seen as the moon's rays strike the squid, protecting the squid from its predators. The squid in turn, provides the bacteria with shelter and a stable source of nutrients.

\section{Conclusion}

As a result, the process of convergence between art and science plays an important role to drive a better discussion and as equal partners in compromising the concept of interdisciplinary, and collaborative activities. 
Furthermore, one's can perceive a world where artists and scientists collaborate directly with each other to create innovative interdisciplinary work, where it carries a positive impact on society, technology and culture. Further, how to increase recognition of interaction in various fields of knowledge, thinking and communication systems. Where all knowledge system and artistic theories are perceived in a light of a new learning culture and can transforms visual art from object making to processes of creating beyond the normal visual sensation. Create an exciting development and sharing experience in producing a creative activities within various disciplines, as well as a visually stunning and celebratory one between people, space, places and all living creatures. Perhaps it creates the attraction within scientists and artists for beings actively in seeking and aiming to articulate the intangible world. In which, both scientists and artists are off making systematic and testable hypotheses about the world and its creation environment. Seeing the magical creation and mysteries, all about the binaries and coupling: left and right brain, thought versus emotion, reason versus intuition, ideas and vision, exploring and experiencing. Which addresses and revealed the meaning, form and function from how the research materials being digested. In order to provide a better contribution to the process of art making into contexts, such as to social-culture, aesthetic, ecological, environment and economic benefit, particularly to the development of Malaysian contemporary art.

\section{References}

Lupton, D, 1992, Australian Journal of Public Health 16(2):145-50

Okpoluaefe Godspower Udezi,S.O., Parameswaran, K., Baharin, H., Othman,S.S, and Saad, W.Z., 2017, You light up my life!, edited by Faridah Qamaruz Zaman and Mohd Nasir Baharuddin. LIGHT - Nature's Yield and Wonders Of Art(NYAWA), 2017, UPM Press, University Putra Malaysia.

Roslan. S., Ghiami, Z., Ahmad, N., and Dangko,J., 2016, Word Illumination, edited by Faridah Qamaruz Zaman and Mohd Nasir Baharuddin. BRAIN - Nature's Yield and Wonders Of Art(NYAWA), 2016, UPM Press, University Putra Malaysia.

Wilson, S., 2010, Art and Science Now, (Thames and Hudson Ltd, London).

Wilson, S., 2008, Beyond the Digital: Preparing artists to work at the frontiers of technoculture. In: Organizations: Multiple Agents with Multiple Criteria, edited by M. Alexenberg. Educating artist for the future, Learning at the intersections of Art, Science, Technology and Culture, 2008, (Intellect Books, The University of Chicago Press, USA), pp. 29-44.

Einstein, A., Mae. C. J., Wilson. E, O., 2018, Exhibition text for Art Science Museum, Art Science Museum Singapore.

Interaction - Science and Art: Divergence and Convergence by (Michal Giboda, 2005), Date of access: 10.10.2018

http://www.sciart-cz.eu/pdf/interaction.pdf.

Oxford living Dictionaries, Date of access: 10.10.2018

https://en.oxforddictionaries.com/definition/technoculture. 